\title{
Nódulo proliferativo en nevus melanocítico congénito gigante
}

\section{Proliferative nodule in giant congenital melanocytic nevus}

\author{
Autores: Celeste Andrea Lorefice ${ }^{1}$, Andrea Isabel Arza \\ Médico a cargo: Patricia Monteleone ${ }^{2}$ \\ Director de Curso: Mirta Moreno ${ }^{3}$
}

\section{RESUMEN}

El nevus melanocítico gigante es una entidad poco frecuente. En los primeros meses o años de vida, pueden aparecer nódulos dérmicos pequeños o grandes, muy pigmentados, que pueden crecer rápidamente o incluso ulcerarse. Esto obliga a realizar diagnóstico diferencial con el melanoma. Se presenta el caso de una paciente de 3 años de edad, con gran lesión pigmentada en pierna izquierda, con nódulos de rápido crecimiento, compatibles con nódulo proliferativo.

Palabras claves: nevus melanocítico gigante, nódulo proliferativo.

\section{ABSTRACT}

Giant melanocytic nevi are rare. In the first few months or even years of life, they may develop small or large dermic nodules, very pigmented, with rapid growth o even ulcer formation. This forces the differential diagnosis with melanoma. We present a case of a 3 year old female patient, with a large pigmented lesion on the left leg, with nodules compatible with proliferative nodules.

Keywords: giant melanocytic nevus, proliferative nodule

REVISTA ARGENTINA DE CIRUGÍA PLÁSTICA 2020;26(1):31-35. HTTPS://DOI.ORG/10.32825/RACP/202001/0031-0035

\section{INTRODUCCIÓN}

Se define como nevo melanocítico congénito (NMC) a las lesiones que están presentes al momento del nacimiento o aparecen en las primeras semanas de vida. Se desarrollan entre las semanas 5 y 25 de la gestación. Los melanoblastos migran desde la cresta neural a varios sitios del cuerpo que incluyen la piel, membranas mucosas, leptomeninges, mesenterio, ojos y orejas. Luego de la migración a estas localizaciones anatómicas entre las semanas 8 y 10 de la gestación, los melanoblastos se diferencian en melanocitos dendríticos. El crecimiento desregulado durante el desarrollo neuroectodérmico en cualquier punto de la migración y diferenciación resulta en la formación de un nevus melanocítico congénito.

Pueden estar presentes en cualquier parte del cuerpo y tener un gran espectro de colores (varios tonos de marón, negro, rosa) y cambios topográficos, como presencia de vellos, nódulos y pápulas o superficie verrucosa o mamelonada. Las potenciales complicaciones de

1. Cursista de 3er año de Curso Superior de Cirugia Plástica de SACPER

2. Médica a cargo

3. Directora de Curso

$\triangle$ Correspondencia: revista@sacper.org.ar

Los autores no declaran conflictos de intereses

Recibido: 25/10/2019/ Aceptado: 07/12/2019 los NMC incluyen el desarrollo de melanoma y melanosis (afectación del sistema nervioso central).

Los NMC pequeños y medianos son comunes, mientras que los grandes y gigantes son raros, se ven en 1/20.000- $1 / 50.000$ recién nacidos.

Ha habido varias definiciones para el nevus "gigante”, incluyendo afectación de una porción significante de un área anatómica (cara o mano), nevus que miden más de $20 \mathrm{~cm}$ o más de diámetro, nevus que cubren más de $1 \%$ del área corporal total en la región de cabeza y cuello o $2 \%$ del área corporal total en el resto del cuerpo. Bittencourt define "gigante" como un nevus melanocítico que mide $20 \mathrm{~cm}$ o más en el adulto, lo

TABLA 1. Características histológicas del nódulo proliferativo en el NMC

\begin{tabular}{|l|l|}
\hline Macroscópico & $\begin{array}{l}\text { Pequeño (menor de } 5 \mathrm{~mm} \text { ) } \\
\text { Nodular } \\
\text { Superficie lisa (ulceración rara) } \\
\text { Marrón oscuro a negro } \\
\text { Múltiple o único }\end{array}$ \\
\hline Microscópico & $\begin{array}{l}\text { Nodular, agregados celulares cohesivos en } \\
\text { dermis } \\
\text { Células más grandes que las asociadas a ne- } \\
\text { vus } \\
\text { Células mezcladas con las asociadas a nevus } \\
\text { Falta de interfaz abrupta con nevus adya- } \\
\text { cente } \\
\text { Macronucleolos } \\
\text { Bajo índice mitótico } \\
\text { Poca o ninguna inflamación } \\
\text { Hemorragia } \\
\text { Sin necrosis (excepto ulceración) } \\
\text { Sin crecimiento destructivo }\end{array}$ \\
\hline Evolución sin resección & $\begin{array}{l}\text { Ablandamiento } \\
\text { Falta de crecimiento progresivo } \\
\text { Disminución de tamaño y pigmentación }\end{array}$ \\
\hline
\end{tabular}




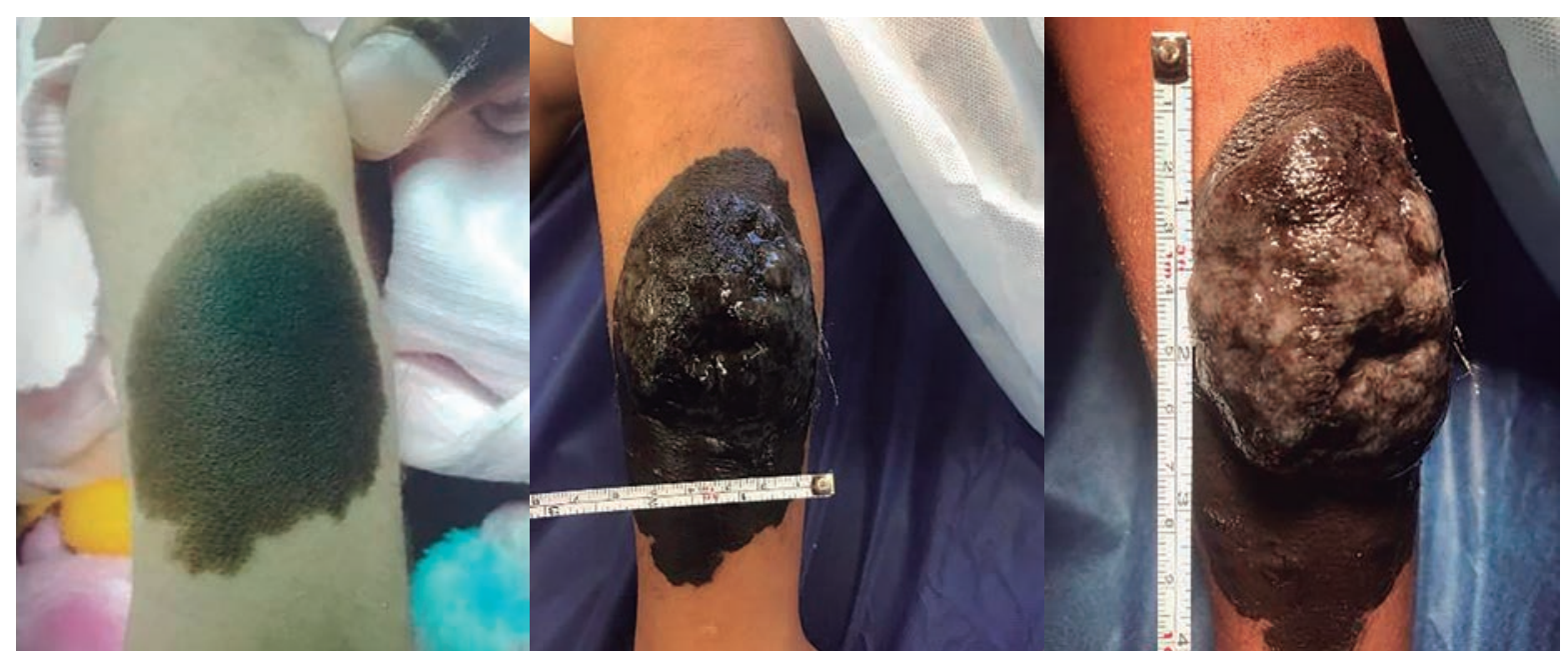

Figura 1. Evolución de la lesión en el lapso de 1 año, con aparición de un nódulo rugoso y ulcerado en su superficie.

que se traduciría en un estimado de $9 \mathrm{~cm}$ en la cabeza de un niño o $6 \mathrm{~cm}$ en el cuerpo de un infante ${ }^{1}$.

Los NMC gigantes tienden a cubrir segmentos del tronco, extremidades o de cabeza y cuello. El mecanismo subyacente de estos patrones es desconocido, pero probablemente represente el patrón embriológico de migración de los melanocitos y el momento de la mutación durante el desarrollo. El crecimiento de un NMC grande es proporcional al crecimiento del niño. La coloración, nodularidad, rugosidad y vellosidad pueden no ser estáticos y a menudo evolucionan con el tiempo. Muchos pacientes sufrirán xerosis y eczema en áreas del NMC, debido a un número reducido o inefectividad de glándulas sebáceas. También se ha reportado aumento de prurito en una porción significante de pacientes.

El gran tamaño es el mayor factor de riesgo para el desarrollo de melanoma para pacientes con NMC, especialmente en nevus de 40 a $60 \mathrm{~cm}$. El melanoma surge de la piel y tejido subcutáneo. A diferencia del melanoma convencional, el de los pacientes con NMC grandes tiende a ocurrir en la primera década de la vida, a menudo antes de la pubertad ${ }^{2}$.

\section{DIAGNÓSTICO}

Aunque el diagnóstico de NMC grandes o gigantes es obvio al nacimiento, algunas características potencialmente alarmantes pueden aparecer en los primeras semanas o meses. Los nódulos proliferativos (NP) son nódulos benignos o pápulas presentes en la dermis, que pueden causar sospecha de melanoma.

Aunque clínicamente son inquietantes, pueden ser clínica e histológicamente benignas. El comportamiento de estas lesiones puede seguir dos patrones:

$\mathrm{NMC}$ con nódulos dérmicos pequeños (menor de $1 \mathrm{~cm}$ de diámetro). A menudo están presentes en el nacimiento pero pueden aparecer a lo largo de la vida, la mayoría durante la infancia.

Nódulos dérmicos grandes (mayores de $1 \mathrm{~cm}$ ). Son menos frecuentes, pueden crecer de forma rápida inicialmente, ocasionalmente ulcerarse y a menudo están muy pigmentados. Si se dejan sin tratamiento, a menudo se hacen más pequeños y más blandos, y no exhiben un fenotipo maligno.

Dentro de estos dos patrones clínicos, la apariencia histológica puede variar (Tabla 1). Los nódulos dérmicos más grandes pueden contener varios tipos de células debido al desarrollo embriológico de los melanocitos a partir de la cresta neural. El rango de apariencia histológica incluye a los siguientes:

- Nódulos proliferativos benignos o expansivos: focos de alta celularidad o nódulos de melanocitos atípicos.

- Nódulos dérmicos y subcutáneos: pueden estar compuestos por melanocitos pequeños, monomorfos, epitelioides y pueden presentar diferenciación neural $\mathrm{o}$, más raro, mesenquimática ${ }^{3}$.

\section{MELANOMA VS. NÓDULO PROLIFERATIVO}

El riesgo a lo largo de la vida de desarrollo de melanoma en NMC es entre el 5 al 10\%, con la mayoría de los casos reportados que ocurren en los primeros 5 años de vida. La mayoría de los melanomas ocurren en los tejidos blandos profundos, mientras que el melanoma que aparece en la epidermis o dermis son considerablemente menos frecuentes. Los NP ocurren con mayor frecuencia en la dermis que los melanomas letales. Especialmente en los NMC gigantes, en que los NP son relativamente comunes, así como en los de mediano tamaño.

En los nevus congénitos pequeños, en los que es más raro encontrar NP, hay una proporción mayor de melanomas superficiales que involucran la epidermis o dermis en comparación con NP. Cuando los melanomas surgen de NC pequeños, tienden a ser más superficiales, opuesto a los melanomas más profundos que se encuentran en los NMC gigantes. Hay muy pocos casos de melanoma letal o metastásico que aparezcan fuera del tejido profundo. 


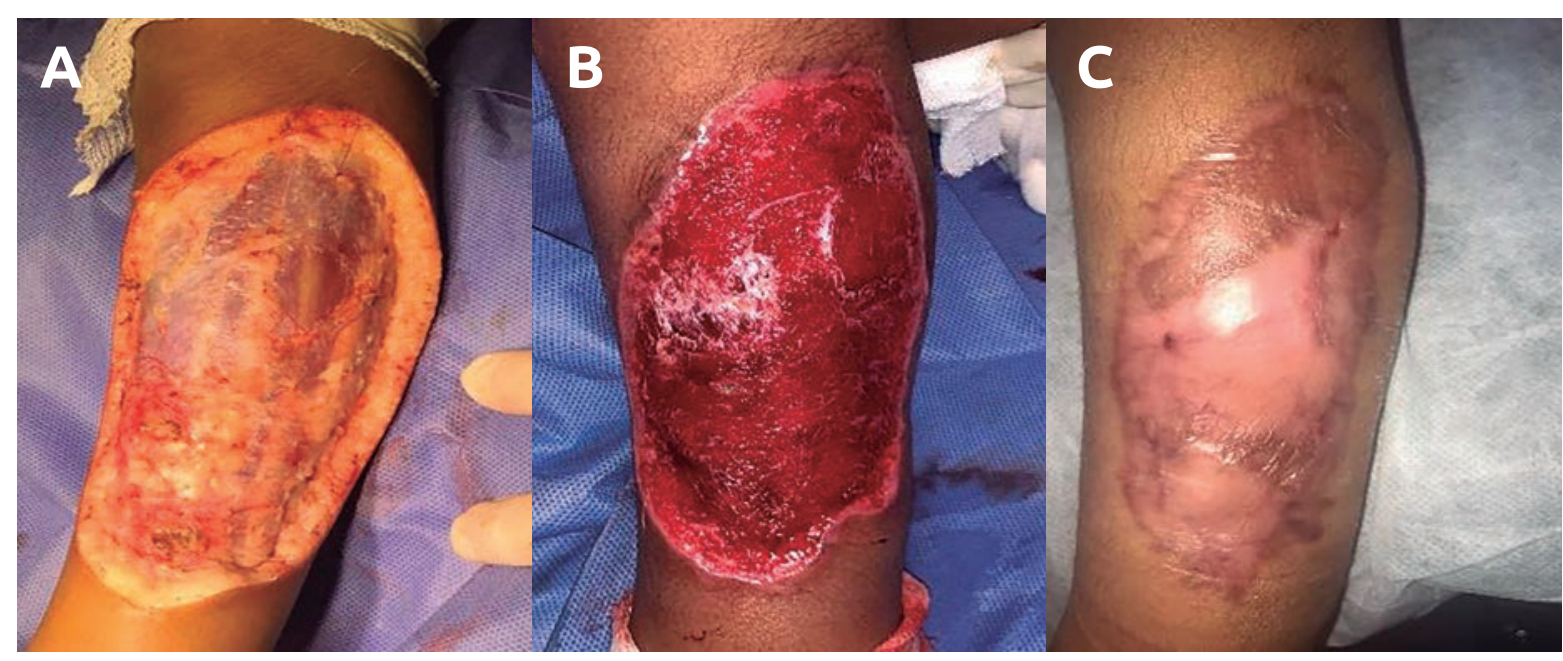

Figura 2. A. Resección de lesión hasta fascia. B. 14 días POP: se observa lecho granulante apto para ser injertado. C. Resultado alejado de injerto de piel parcial

Clínicamente, la multiplicidad de las lesiones favorece el diagnostico de NP, mientras que la ulceración es infrecuente en los PN en comparación con los melanomas letales. ${ }^{4}$

El diagnóstico correcto es crucial, ya que los pacientes con NP no suelen tener riesgo aumentado de melanoma. La inmunohistoquímica tiene valor limitado para diferenciar los NP del melanoma. La hibridación fluorescente in situ (FISH) y la hibridación genómica comparativa pueden ser de utilidad para el diagnóstico de proliferaciones melanocíticas ambiguas. La alta densidad celular de los NP contrasta con el nevus congénito que lo rodea. Los NP atípicos tienen un índice de Ki67 más alto que el NMC que lo rodea, por lo que no puede ser usado para diferenciarlo del melanoma. Pero en población pediátrica, ni el FISH ni IHQ son de ayuda para distinguir los NP del melanoma infantil, en contraste con el melanoma de adulto asociado con $\mathrm{NMC}^{5}$.

\section{TRATAMIENTO}

El tratamiento debe ser abordado en forma multidisciplinaria y en etapas tempranas. Las indicaciones para la escisión quirúrgica incluyen: casos de melanoma establecido, escisión profiláctica para disminuir el riesgo de la degeneración maligna, reducción y control de los síntomas, mejoría estética, bienestar psicológico y mantenimiento de función, el beneficio psicológico del tratamiento en edad preescolar, aumento de la elasticidad de los tejidos en este grupo etario ${ }^{6}$.

Se han descrito varias opciones de tratamiento no quirúrgico, como la dermoabrasión, ablación por láser, curetaje y peeling químico. Estas modalidades pueden disminuir la carga de células del nevus, pero no resultan en una eliminación completa de las células, y terminan en una falta de diagnóstico histológico y dificultad en el monitoreo posoperatorio en búsqueda de cambios clínicos. En contraste, la escisión directa del

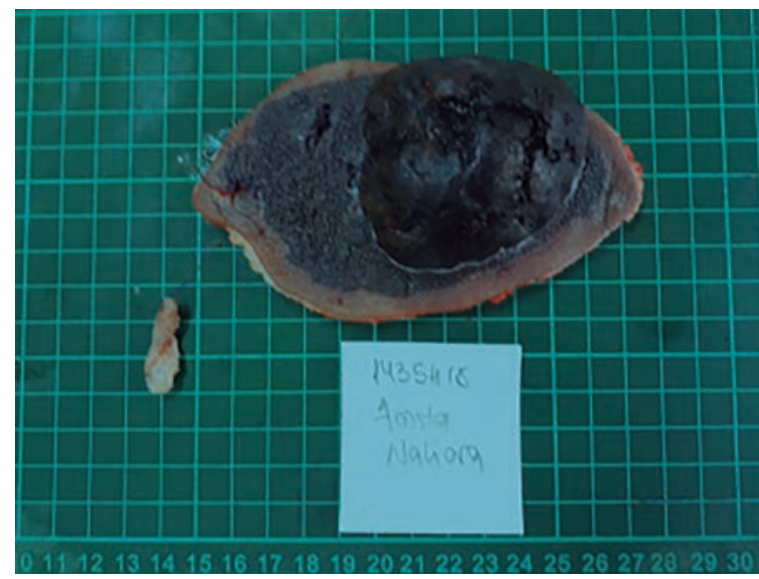

Figura 3. Anatomía patológica. Losange cutánea de 12,5×7 cm, con lesión de color negruzco, bordes irregulares pero bien definidos. Se observa en su interior crecimiento nodular de 5,5×5x2 cm con superficie de aspecto erosionado. Los cortes histológicos muestran piel con proliferación celular melanocítica organizada en nidos y playas que se extiende desde la unión dermoepidérmica hasta la hipodermis rodeando estructuras vasculares y anexos cutáneos. Muestra en su superficie erosión con proliferación vascular.se encuentra constituida en parte por células de núcleos redondeados de citoplasma con abundante pigmento melánico. Presenta sectores donde los melanocitos adquieren mayor tamaño con núcleos de leve atipia, presencia de nucléolos de gran tamaño con citoplasma eosinófilo mal delimitado y variable pigmento melánico, con algunas mitosis típicas, sin necrosis. Tumor melanocítico atípico que asienta dentro de un nevo melanocítico congénito.

nevus es actualmente la opción terapéutica más aceptada para los nevus melanocíticos congénitos gigantes. Hay varias opciones quirúrgicas para la reconstrucción del defecto resultante de esta escisión. La más simple de las opciones es la escisión seriada y cierre directo del defecto en etapas. Sin embargo, si el defecto no puede ser cerrado luego del avance cutáneo, otras opciones incluyen injerto de piel total o parcial, expansión tisular, substitutos cutáneos y transferencia libre de tejido 7 . Un seguimiento continuo es imperativo luego del tratamiento, ya que muchos pacientes pueden tener már- 
genes positivos y/o nevus satélites que deben ser monitoreados por signos de transformación maligna.

\section{MATERIALES Y MÉTODOS}

\section{CASO CLÍNICO}

Paciente femenino de 3 años de edad, que presenta nevus en miembro inferior izquierdo desde el nacimiento. Al momento de la consulta lesión pigmentada, de $12 \times 6 \mathrm{~cm}$ que presenta tumoración exofítica, dolorosa, pruriginosa con signos de infección de 1 año de evolución, compatible con nódulo proliferativo $v$ s. melanoma (Figura 1).

Presenta biopsia previa: nevo melanocitico con rasgos congénitos.

Se decide la escisión total de la lesión hasta la fascia, con margen de seguridad de $1 \mathrm{~cm}$.

Se realiza curación con gasas de propóleo hasta lograr un lecho granulante.

A los 14 días se realiza injerto de piel parcial, zona dadora muslo ipsilateral, logrando un resultado cosmético aceptable.

Dada las características histológicas y la edad de la paciente se asume esta entidad como nódulo proliferativo benigno y se realiza seguimiento cercano del paciente (Figuras 2 y 3 ).

\section{DISCUSIÓN}

El desarrollo de proliferaciones nodulares focales dentro de un NMC grande puede simular un melanoma maligno nodular. A pesar de su apariencia clínica alarmante, los nódulos proliferativos (NP) a menudo son benignos, pueden remitir y son fenotípicamente benignos en modelos experimentales. Algunos NP y grandes NMC muestran rasgos histológicos atípicos, lo que hace más difícil diferenciarlo de un melanoma maligno. El gran tamaño es el mayor factor de riesgo para el desarrollo de melanoma para pacientes con NMC, especialmente en nevus de $40 \mathrm{a} 60 \mathrm{~cm}$, cuando presentan localización en tronco, numerosos nevus satélites. Es menos frecuente que ocurra en pacientes con NMC limitados a la cabeza y cuello o una extremidad. El melanoma surge de la piel y tejido subcutáneo. A diferencia del melanoma convencional, el de los pacientes con NMC grandes tiende a ocurrir en la primera década de la vida, a menudo antes de la pubertad.

El riesgo de melanoma ha sido sobreestimado en las últimas décadas en la literatura, ya que aunque aproximadamente la mitad de los melanomas que suceden en pacientes con grandes NMC ocurren dentro de los primeros 5 años de vida, la presentación de nódulos proli- ferativos con comportamiento clínico benigno y características histológicas benignas que simulan melanomas pueden surgir en la infancia y potencialmente llevar a una sobreestimación de la probabilidad de malignidad en este grupo etario.

El tratamiento quirúrgico puede ir desde dermoabrasión, curetaje, laser, expansión tisular con escisión seriada, o uso de flaps o injertos. Suele ser imposible quitar cada célula del nevus en grandes lesiones, debido al gran tamaño y la afección de estructuras más profundas como grasa, fascia e incluso músculo. Se han reportado casos de melanoma desarrollado por debajo de injertos de piel en pacientes luego de la escisión "completa" de un gran NMC. Aunque varios estudios han demostrado una tendencia a una menor incidencia de melanoma en pacientes cuyos nevus fueron parcialmente o totalmente removidos, suelen ser los nevus más grandes, que tienen mayor riesgo de melanoma, pero que a su vez es más probable que sean inoperables 8 .

Al día de la fecha, no hay evidencia de que la remoción de grandes o gigantes NMC reduzca el riesgo de melanoma.

\section{CONCLUSIÓN}

El manejo clínico y la interpretación de proliferaciones atípicas en grandes NMC presentan desafíos a dermatólogos y patólogos. Los NMC de más de $10 \mathrm{~cm}$ de diámetro tienen un mayor riesgo de transformación maligna y melanoma maligno. En la práctica, un cambio pigmentario visible o aparición de nódulos dentro de un NMC debe seguirse con una evaluación histopatológica para descartar melanoma. Por eso es importante el seguimiento estrecho del paciente con nevus melanocítico congénito grande/gigante, y ante sospecha de melanoma o diagnóstico confirmado del mismo, está indicada su resección para obtener un diagnóstico histopatológico completo. La opción terapéutica ideal es la escisión completa, en una o varias etapas y luego reconstrucción con colgajos locales, expansión o cobertura con injertos cutáneos o sustitutos dérmicos para lograr un resultado estético aceptable. En el caso expuesto en este trabajo, la paciente presentó cambios morfológicos abruptos en su nevus congénito gigante, con aparición de tumoración exofítica, dolorosa, pruriginosa con signos de infección. Estos cambios obligaron a descartar el diagnostico de melanoma con la escisión completa. Debido al tamaño y localización del mismo, se realizó una resección completa de la lesión y cobertura con injerto de piel diferido, logrando un resultado estético aceptable, con baja morbimortalidad. 


\section{BIBLIOGRAFÍA}

1. Arneja J, Gosain A. Giant congenital melanocytic nevi. PRS Journal 2006; Volume 120, Number 2. 2006.

2. Price H. Congenital melanocytic nevi:update in genetics and manag ment. Curr Opin Pediatr 2016, 28:476-82.

3. Leech S, Bell H. Neonatal giant congenital nevi with proliferative nodules. Aclinicopathologic study and literature review of neonatal melanoma. Arch Dermatol 2004;140:83-8.

4. Yelanos $O$, Arva N, Obregon R. A comparative study of proliferative nodules and lethal melanomas in congenital nevi from children. Am J Surg Pathol 2015;39:405-15.

5. Proliferative nodules vs melanoma arising in giant congenital me lanocytic nevi during childhood. Beatrice Vergier; ELodieLaharanne;
Martina Prochazlova. JAMA Dermatol. doi:10.1001/jamaderma tol.2016.2667. Published online August 3, 2016

6. Proliferative nodules in a giant congenital melanocytic nevus-case report and review of the literatura. A H van Houten; M C van Dijk; M Schutterlaar. Journal of CutaneousPathology. 2009

7. Proliferative nodules in congenital melanocityc nevi. A clinicopathologic and inmunohistochemical analysis. Mark Herron; Sherryllvanderhooft, Kristi Smock. (Am J SurgPathol 2004; 28:1017.1025)

8. Congenital melanocytic nevi-when to worry and how to treat: Facts and controversies. Harper N. Price, MD, Julie V. Schaffer, MD . Clinics in Dermatology (2010) 28, 293-302 